\title{
Expression of the chemokine CXCL14 in the tumour stroma is an independent marker of survival in breast cancer
}

\author{
Elin Sjöberg ${ }^{1}$, Martin Augsten ${ }^{2}$, Jonas Bergh ${ }^{3}$, Karin Jirström ${ }^{4}$ and Arne Östman ${ }^{*, 1}$ \\ ${ }^{1}$ Department of Oncology-Pathology, Cancer Center Karolinska (CCK), Karolinska Institutet, 17176 Stockholm, Sweden; ${ }^{2}$ Division \\ for Vascular Oncology and Metastasis, German Cancer Research Center (DKFZ), 69120 Heidelberg, Germany; ${ }^{3}$ Department of \\ Oncology-Pathology, Cancer Center Karolinska (CCK), Karolinska Institutet and Radiumhemmet-Karolinska Oncology, Karolinska \\ University Hospital, 17176 Stockholm, Sweden and ${ }^{4}$ Division of Oncology and Pathology, Department of Clinical Sciences, Lund \\ University 221 85, Lund, Sweden
}

Background: Expression of the chemokine CXCL14 has previously been shown to be elevated in the tumour stroma of, for example, prostate and breast cancer. Cancer-associated fibroblast-derived CXCL14 enhances tumour growth in mouse models of prostate and breast cancer. However, the prognostic significance of compartment-specific expression of CXCL14 has not been studied.

Methods: CXCL14 mRNA expression was analysed in a breast cancer tissue microarray (TMA) of formalin-fixed, paraffinembedded tumours by the RNAscope 2.0 Assay. Epithelial and stromal expression was analysed separately and correlated with clinicopathological characteristics and survival.

Results: CXCL14 was variably and independently expressed in malignant and stromal cells of breast cancer. Total and stromal expression of CXCL14 did not associate with clinicopathological parameters. Epithelial CXCL14 expression was significantly associated with oestrogen receptor $\alpha(E R \alpha)$-positive tumours and lower proliferation status. Total CXCL14 expression correlated significantly with shorter breast cancer-specific and recurrence-free survival. High stromal, but not epithelial, CXCL14 expression was significantly associated with shorter survival in univariable and multivariable analyses. Moreover, the correlation between stromal CXCL14 expression and survival was more prominent in ER negative, triple negative and basal-like breast cancers.

Conclusions: The identification of prognostic significance of stromal CXCL14 in breast cancer demonstrates novel clinical relevance of a stroma-derived secreted factor and illustrates the importance of tumour compartment-specific analyses. On the basis of the prognostic signals from difficult-to-treat subgroups, CXCL14 should also be considered as a candidate drug target.

Tumours develop and progress through an interplay between cancer cells and the stroma. The tumour stroma or tumour microenvironment (TME) harbours many different cell types including endothelial and lymphatic cells, pericytes, various immune cells and fibroblasts embedded in an extracellular matrix (ECM). Both the epithelial cancer compartment and the TME produce a plethora of soluble factors, different types of vesicles, matrix components and matrix-modifying enzymes that promote cancer cell survival, migration, invasion and dissemination (Pietras and Ostman, 2010; Goubran et al, 2014).
Among the secreted factors involved in the paracrine tumour cross-talk are chemokines; small proteins of about $8-14 \mathrm{kDa}$ that act both in an autocrine and in a paracrine manner and exert local and systemic effects. Chemokines create a gradient along which cells, expressing the corresponding receptors, travel to the source of this gradient. Thus, tumour-derived chemokines have a crucial role in the recruitment of different cell types into the tumour and in their subsequent activation. Chemokines have been demonstrated to exert potent pro- and anti-tumorigenic effects, depending on the context. For example, certain chemokines stimulate tumour growth

*Correspondence: Professor A Östman; E-mail: arne.ostman@ki.se

Received 4 February 2016; revised 9 March 2016; accepted 21 March 2016; published online 26 April 2016

(C) 2016 Cancer Research UK. All rights reserved 0007-0920/16 
and invasion of cancer cells and promote angiogenesis, while other chemokines inhibit angiogenesis by sequestering pro-angiogenic factors (Orimo et al, 2005; Strieter et al, 2006; Augsten et al, 2009). Chemokines also mobilise and recruit different bone marrowderived cells and activate stromal cells in sites of inflammation, primary tumours or metastasis (Balkwill, 2004; Lazennec and Richmond, 2010).

The orphan chemokine CXCL14, earlier designated BRAK, MIP- $2 \gamma$, BMAC or KS1, displays chemotactic activity towards immature dendritic cells, monocytes, macrophages and NK cells but not $\mathrm{T}$ cells (Sleeman et al, 2000; Kurth et al, 2001; Shellenberger et al, 2004; Shurin et al, 2005; Starnes et al, 2006). Enhanced expression of CXCL14 has been found in different types of cancer, including tumours arising in colon, pancreas, prostate and breast, where CXCL14 is expressed by cancer cells or stromal cells or by both cell types (Allinen et al, 2004; Schwarze et al, 2005; Wente et al, 2008; Williams et al, 2014). However, in lung cancer CXCL14 is epigenetically silenced, and re-activation of CXCL14 expression in tumour cells suppressed tumour growth in xenograft models (Tessema et al, 2010). As other chemokines, CXCL14 has shown context-dependent pro- or anti-tumoral effects in experimental models systems, as recently discussed by Hara and Tanegashima (2012). Some findings suggest that the net-effects of CXCL14 on tumour growth depend on the cell type expressing this factor. For example, in animal models CXCL14 promotes the growth of breast and prostate tumours when expressed by stromal fibroblasts but shows anti-tumoral effects when expressed by the malignant cells (Allinen et al, 2004; Schwarze et al, 2005; Gu et al, 2012; Augsten et al, 2014). The tumour stimulatory effects of stroma-derived CXCL14 are suggested to include pro-angiogenic effects and its ability to recruit monocytes to tumours (Augsten et al, 2009). Notably, the clinical significance of these findings remains to be further characterised by high-resolution analysis of CXCL14 expression in human tumour samples.

There are only a few studies so far that have investigated the potential prognostic value of CXCL14 expression in cancer, by analysing protein expression in whole tumour tissue using immunohistochemistry (IHC). Most of these analyses demonstrated that high CXCL14 protein levels in tumours correlate with a more favourable prognosis ( $\mathrm{Gu}$ et al, 2012; Lin et al, 2014; Sand et al, 2015). Importantly, these earlier IHC studies have not addressed the possibility that the prognostic impact of CXCL14 may be determined by the tumour compartment in which CXCL14 is produced.

In light of these experimental and early clinical findings this study aimed at characterising, in a compartment-specific manner, CXCL14 expression in human breast cancer and to explore potential links to clinicopathological characteristics and outcome.

\section{MATERIALS AND METHODS}

Paraffin embedding of cultured fibroblasts and xenograft tumours. Previously established fibroblasts overexpressing CXCL14 (NIH-CXCL14) and control fibroblasts (NIH-ctr) (Augsten et al, 2009) were cultured in DMEM supplemented with $10 \%$ fetal calf serum (Sigma-Aldrich, Stockholm, Sweden), 1\% glutamine and antibiotics (penicillin (100 units per $\mathrm{ml}$, Sigma-Aldrich) and streptomycin $\left(100 \mu \mathrm{g} \mathrm{ml}^{-1}\right.$, Sigma-Aldrich)). Equal number of cells were harvested, centrifuged at 1500 r.p.m. for $5 \mathrm{~min}$ and fixed overnight with $4 \%$ paraformaldehyde (Sigma-Aldrich). Xenograft tumours from co-injection experiments of MCF7 cells and NIHCXCL14 fibroblasts or NIH-ctr fibroblasts into SCID mice have been described previously (Augsten et al, 2014).

Cell pellets and xenograft tumours were placed in tissue embedding boxes in $70 \%$ ethanol and dehydrated using increasing percentages of ethanol, followed by paraffin embedding and sectioning on Superfrost Plus slides (Menzel-Gläser, Braunschweig, Germany).

Tumour collection and tissue microarray. The breast cancer tissue microarray (TMA) was made from a consecutive series of tumours from 498 primary breast cancer patients diagnosed at the Department of Pathology, Malmö University Hospital between 1988 and 1992. Tissue was prepared and fixed in 10\% natural buffered formalin (NBF) for 6-72 h. The median age at diagnosis was 64.2 years (range, 27-96 years), and follow-up data on recurrence-free survival and breast cancer-specific survival were available for 491 and 494 patients, respectively. The median followup time for patients without breast cancer event was 106 months (range, 0-207 months). Information on adjuvant therapy was available for 389 patients, 157 of these had received adjuvant tamoxifen, 19 adjuvant chemotherapy and 4 patients had received a combination of tamoxifen and chemotherapy. In all, 209 patients did not receive any adjuvant treatment. This cohort is described in detail elsewhere (Borgquist et al, 2008). Tumours were classified as belonging to the subtype Luminal A, Luminal B, HER2 and Basallike according to the immunohistochemical expression of oestrogen receptor $\alpha(\mathrm{ER} \alpha)$, progesterone receptor (PR) and epidermal growth factor receptor (EGFR). Tumours negative for ER $\alpha$, PR and HER2 were defined as triple negative breast cancers (TNBC). The tumour stroma abundance was determined by semi-quantative scoring of hematoxylin and eosin-stained sections.

CXCL14 RNAscope 2.0 Assay. The RNAscope 2.0 Assay, with a CXCL14-specific probe (Advanced Cell Diagnostics, Hayward, CA, USA), was used for detection of CXCL14 mRNA on the breast tumour TMA. A detailed description of the RNAscope 2.0 Assay has been published before (Wang et al, 2012). In short, tissue sections were deparaffinised in xylene followed by dehydration with $100 \%$ ethanol. The sections were air-dried and incubated with pretreatment 1 solution for $15 \mathrm{~min}$ at room temperature and washed with distilled water. Pretreatment was continued by boiling the slides in pretreatment buffer 2 for $15 \mathrm{~min}$, and the slides were immediately washed with distilled water, followed by $100 \%$ ethanol and then air-dried. Pretreatment buffer 3 was added to the tissue sections, and incubation was performed in a humidity chamber at $40{ }^{\circ} \mathrm{C}$ for $30 \mathrm{~min}$. Slides were washed in distilled water and hybridisation was initiated. The CXCL14 probe (Advanced Cell Diagnostics) was added, and the slides were hybridised in a humidity chamber at $40^{\circ} \mathrm{C}$ for $2 \mathrm{~h}$, followed by incubation with Amp1-Amp6. Hybridisation with Amp1 and Amp2 was done at $40{ }^{\circ} \mathrm{C}$ for $30 \mathrm{~min}$ and $15 \mathrm{~min}$, respectively. Amp3 and Amp4 hybridisation was also performed at $40{ }^{\circ} \mathrm{C}$ for $30 \mathrm{~min}$ and $15 \mathrm{~min}$, respectively. The slides were incubated with Amp5 and Amp6 at room temperature for $30 \mathrm{~min}$ and $15 \mathrm{~min}$, respectively. Slides were washed in $1 \times$ wash buffer after each step. Detection was performed using DAB followed by haematoxylin counterstaining (Histolab Products AB, Gothenburg, Sweden), dehydration and mounting with Vectamount permanent mounting media (Vector Laboratories, Inc., Burlingame, CA, USA). The CXCL14 mRNA staining was scored blinded as negative or positive with independent scoring of total tumour tissue, epithelial and stromal compartments.

Statistics. The $X^{2}$ test was used for analysing association of CXCL14 expression with clinicopathological parameters, including age, tumour size, Nottingham histological grade (NHG), lymphnode metastasis (node status), ER $\alpha$-, PR- and HER2 receptor status and tumour cell proliferation (Ki67 expression), and with breast cancer subtypes (Luminal A, Luminal B, Basal and HER2), tumour stroma abundance and adjuvant therapy.

The Kaplan-Meier method and log-rank test were used to estimate recurrence-free survival and breast cancer-specific 
survival. Primary events included in the survival analysis were loco-regional recurrence and distant metastasis for recurrence-free survival, and breast cancer-specific death. Contralateral events and non-breast cancer-specific deaths were excluded.

Cox proportional hazards model was used for comparing hazard ratios (HRs) in both univariable and multivariable analyses. Multivariable analyses included known prognostic- and therapy predictive factors, such as age, tumour size, NHG, node status, ER $\alpha$ - and HER2 receptor status. The results are presented as HRs including their 95\% confidence intervals (CIs). Cox proportional hazards model was further used for analysis of the impact of stromal CXCL14 on survival in different patient subsets.

Two-sided statistical tests were performed and $P$-values below 0.05 were considered as significant. All calculations were performed with the statistical package SPSS 21.0 (SPSS Inc., Chicago, IL, USA).

\section{RESULTS}

CXCL14 is differentially expressed in the stromal and epithelial compartments of breast cancer. To describe CXCL14 expression in human breast tumour tissue, a clinically well-annotated TMA with tumours from 498 patients was stained for CXCL14 mRNA using the RNAscope 2.0 Assay (Wang et al, 2012).

The specificity of the CXCL14 probe (Advanced Cell Diagnostics) was evaluated on sections of paraffin-embedded pellets of fibroblasts overexpressing CXCL14 or control fibroblasts, and on xenograft tumours containing MCF7 breast cancer cells and CXCL14 fibroblasts or control fibroblasts (Supplementary Figure 1), that have been explored in previous studies (Augsten et al, 2009, 2014). For analyses of the clinical samples, CXCL14 staining was scored as negative or positive in total tumour tissue and separately in the epithelial and stromal compartments (Figure 1). Scoring was done blinded with regard to clinicpathological characteristics and outcome.

Of the 498 patient samples represented on the TMA, 394 (79\%) yielded informative staining and were included in the subsequent analyses (Table 1 and Figure 1). Among these patient biopsies, 138 (35\%) were positive for CXCL14 mRNA. Among these, 31 patients $(22 \%)$ showed positivity in both compartments, 48 patients $(35 \%)$ showed staining only in the epithelium and 59 patients $(43 \%)$ displayed positivity only in the tumour stroma. Together, these data demonstrate that breast cancer tissue in general displays variable expression of CXCL14 mRNA, and that expression in the stromal and epithelial compartments is independent.

Epithelial and stromal CXCL14 expression shows different associations with clinicopathological characteristics. To explore the clinical relevance of CXCL14 in breast cancer, associations between clinicopathological characteristics (age, tumour size, NHG, node status, ER $\alpha-$, PR and HER2 receptor status, tumour cell proliferation), breast cancer subtypes (Luminal A, Luminal B, HER2, Basal), adjuvant treatment or tumour stroma abundance and total, epithelial or stromal CXCL14 expression were analysed (Table 1).

Epithelial CXCL14 was significantly associated with ER $\alpha$ positivity $(P=0.006)$ and with decreased proliferation, determined by Ki67 expression $(P=0.049)$ (Table 1$)$. Concerning stromal CXCL14, trends were observed for associations with positive lymph-node status and age $>50$ years. However, neither stromal nor total CXCL14 expression correlated significantly with any tumour or patient characteristics. Notably, stromal CXCL14 expression was not associated with the amount of tumour stroma (Table 1 and Supplementary Table 1).

In summary, epithelial CXCL14 expression is significantly associated with $\mathrm{ER} \alpha$ positivity and low proliferation, whereas stromal CXCL14 expression is not linked to any of the established clinicopathological parameters, subtypes of breast cancer or tumour stroma abundance.

High stromal CXCL14 expression correlates with shorter recurrence-free and breast cancer-specific survival. The results from the CXCL14 expression analyses were combined with patient survival data to explore the potential impact of the marker on outcome. Three sets of independent analyses were performed where breast cancer patients were categorised based on the total CXCL14 expression, the epithelial CXCL14 expression or the stromal CXCL14 expression.

As shown in Supplementary Figure 2, Kaplan-Meier analysis revealed that the group with high total CXCL14 expression displayed a significantly shorter breast cancer-specific survival $(P=0.014)$ and recurrence-free survival $(P=0.028)$. To investigate the impact of compartment-specific expression of CXCL14 on breast cancer outcome, similar analyses were performed for epithelial and stromal expression separately. Of note, high stromal CXCL14, but not epithelial CXCL14, was linked to decreased breast cancer-specific survival $(P=0.001)$ and decreased recurrence-free survival $(P=0.004)$ (Figure 2$)$. Also according to a Cox proportional hazard model, the presence of CXCL14 transcripts in the tumour stroma increased both the risk for breast cancer-

\section{CXCL14 expression}

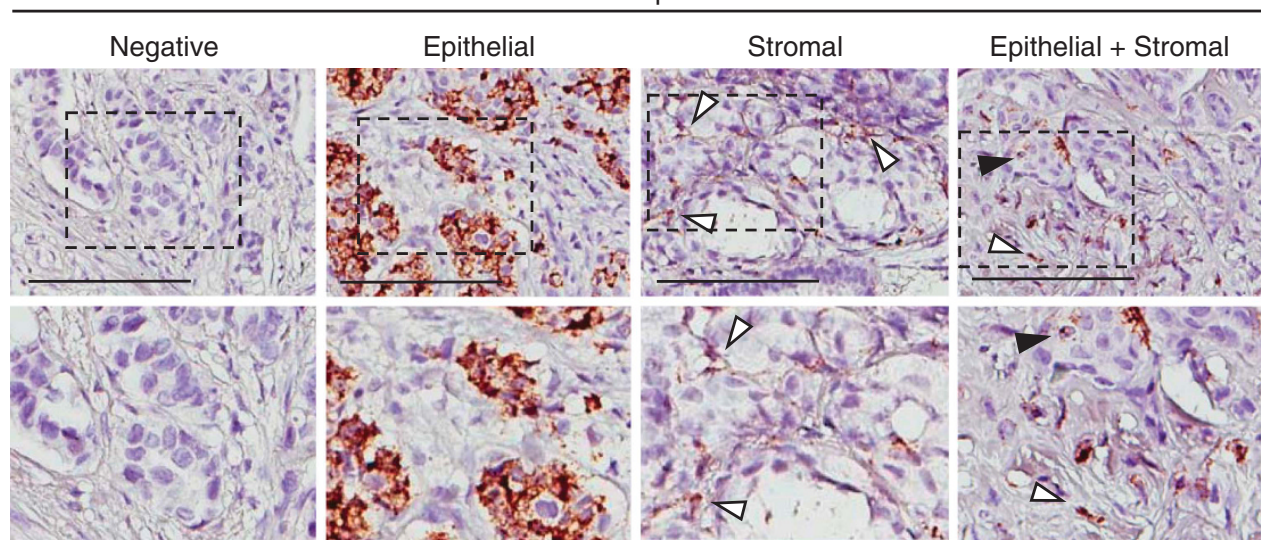

Figure 1. CXCL14 mRNA expression detected with RNAscope in breast tumours. Examples of negative, epithelial, stromal and both epithelial and stromal staining of CXCL14 mRNA in breast tumours. Black arrows show epithelial CXCL14 expression and white arrows show stromal CXCL14 expression (upper panel). Scale bar: $100 \mu \mathrm{m}$. Magnifications of each example (lower panel). 
Table 1. Associations between epithelial or stromal CXCL14 expression and clinicopathological parameters, breast cancer subtypes, adjuvant therapy or stroma abundance

Number (CXCL14 data)

$N=498(394)$

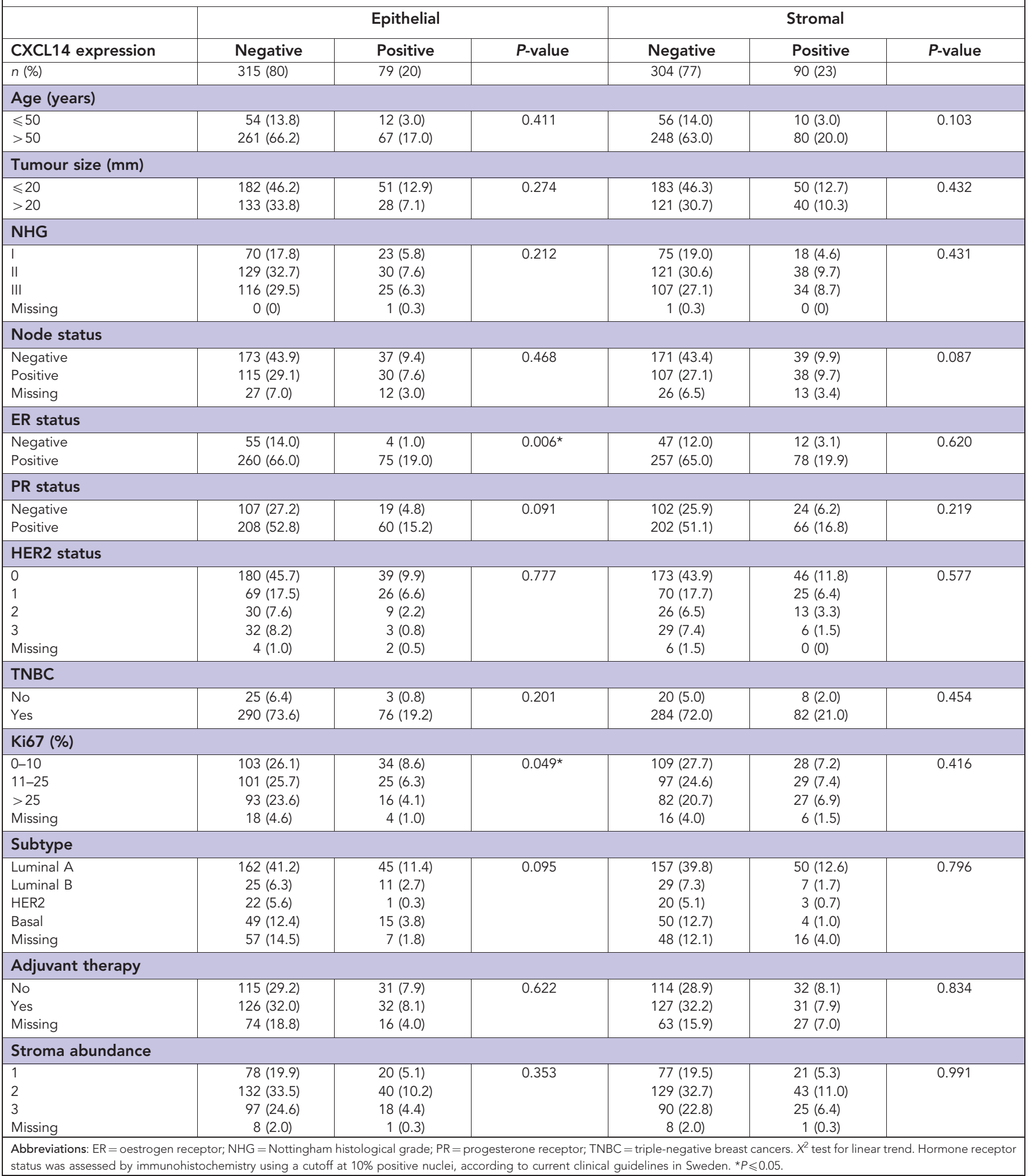

specific death $(\mathrm{HR}=2.22(1.40-3.52)(95 \% \mathrm{CI}))$ and the risk for recurrence $(\mathrm{HR}=1.76(1.19-2.58)(95 \% \mathrm{CI}))$.

To investigate whether the prognostic significance of stromal CXCL14 was particularly strong in certain patient subsets, survival analyses were performed in strata. As shown in Figure 3, the impact of stromal CXCL14 expression on breast cancer-specific survival was particularly prominent in patients with $\mathrm{ER} \alpha$ negative, triple negative and basal-like tumours $(\mathrm{HR}=3.50, \mathrm{HR}=5.08$ and $\mathrm{HR}=3.58$, 

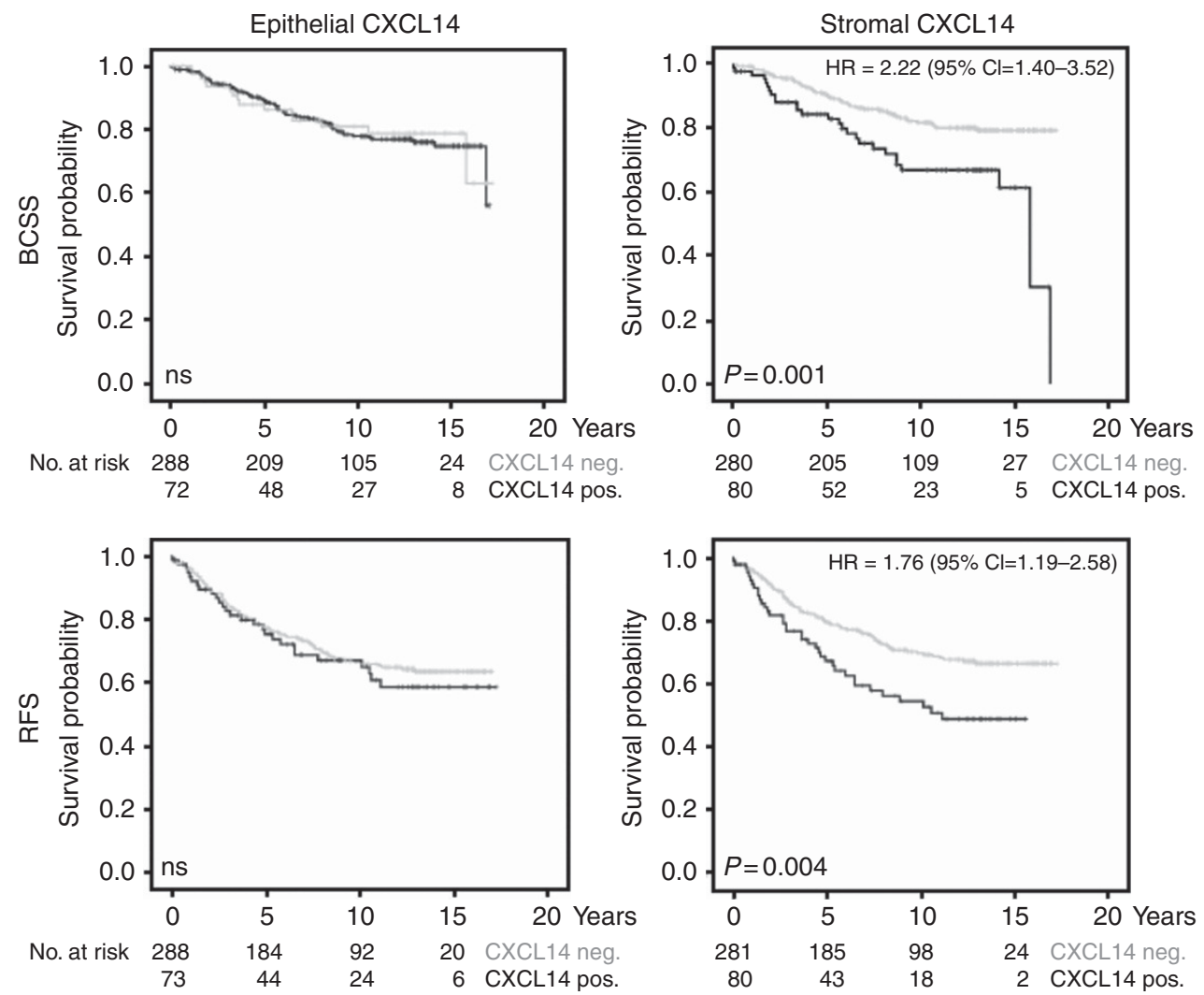

Figure 2. Correlations of stromal CXCL14 expression with worse breast cancer-specific and recurrence-free survival in breast cancer patients. Kaplan-Meier curves for breast cancer-specific survival (BCSS) and recurrence-free survival (RFS) in patients with positive or negative CXCL14 mRNA staining in the tumour stroma (right panel) and in the tumour epithelium (left panel).

respectively). Similar results were obtained in subset analyses using recurrence-free survival as end point (Supplementary Figure 3).

These analyses thus demonstrate significant links between breast cancer survival and total as well as stromal CXCL14 expression. On the basis of explorative sub-set analyses, the survival association for stromal CXCL14 expression was particularly prominent in patients of the $\mathrm{ER} \alpha$ negative-, triple negative and basal subgroups.

CXCL14 expression in the tumour stroma is an independent marker of poor prognosis in breast cancer. The findings of associations between CXCL14 expression and survival prompted further analyses to investigate whether CXCL14 also acts as an independent marker for breast cancer survival. Multivariable analyses were performed including, in addition to CXCL14 status, age, tumour size, NHG, node status, ER $\alpha$ - and HER2 status. Separate analyses were done for total CXCL14 and stromal CXCL14 expression (Table 2 and Supplementary Table 2).

These analyses demonstrated that stromal CXCL14 is an independent marker for breast cancer-specific and recurrence-free survival. Stromal CXCL14 expression was shown to be the third strongest prognostic factor ( $\mathrm{HR}=1.877$ for breast cancer-specific survival), with lymph-node positivity and histological grade as the other factors with a higher HR (Table 2). Analyses based on total CXCL14 yielded similar results $(\mathrm{HR}=1.860$ for breast cancerspecific survival) (Supplementary Table 2). Stromal CXCL14 expression remained significantly associated with survival in multivariable analysis when adjusted for stroma abundance (data not shown).

These analyses thus identify stromal CXCL14 as a candidate novel independent marker for breast cancer survival.

\section{DISCUSSION}

The present study identifies stromal CXCL14 expression as an independent novel marker for decreased breast cancer-specific survival and decreased recurrence-free survival, in particular in $\mathrm{ER} \alpha$ negative-, triple negative and basal-like breast cancers. In contrast, CXCL14 expression in malignant cells did not show a correlation to clinical outcome. These findings add to a series of recent studies demonstrating prognostic significance of stromaexpressed marker proteins and stroma-related gene-signatures (Paulsson and Micke, 2014). Notably, findings have general novelty in the demonstration of prognostic significance of a stromaderived secreted factor.

The findings of associations between stromal CXCL14 expression and an impaired survival are consistent with some earlier studies. CXCL14 was found to be part of gene expression signatures that predict worse disease-free survival of prostate and ovarian cancer (Riester et al, 2014; Williams et al, 2014). In breast cancer, the CXCL14 gene was included in a gene signature that significantly correlated with the presence of clinical metastasis (Chiu et al, 2008). By analysing CXCL14 protein expression, Zeng et al (2013) found the chemokine to be upregulated in colorectal tumour tissue, as compared with normal adjacent colorectal mucosa. In this study, CXCL14 was found to be associated with tumour-node metastasis (TNM) stage, differentiation grade and tumour size, and to correlate with disease recurrence and worse overall survival (Zeng et al, 2013). Other studies have instead reported on associations between high CXCL14 protein expression and good prognosis in breast and colorectal cancer (Gu et al, 2012; Lin et al, 2014). Notably, none of these studies considered the cell type responsible for CXCL14 expression. 


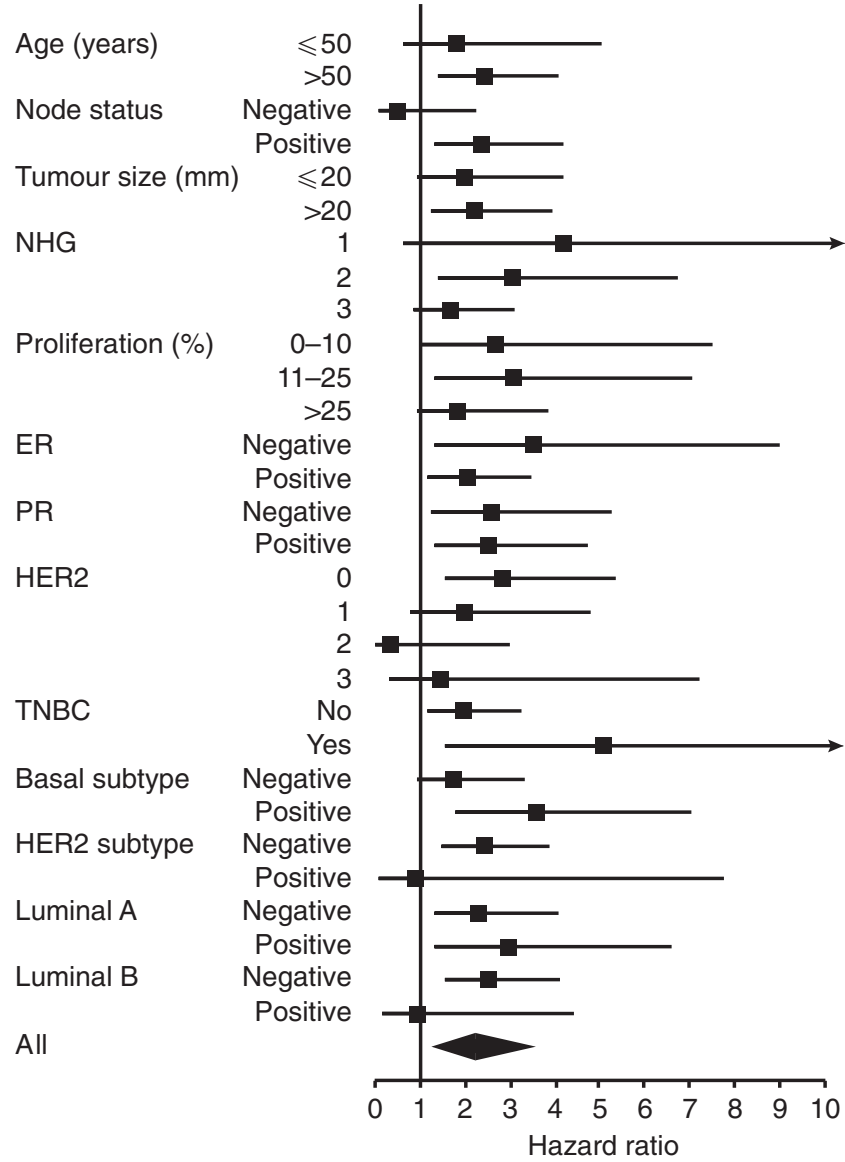

Figure 3. Subgroup-specific analysis of breast cancer-specific survival for stromal CXCL14 in breast cancer patients. A forest plot over hazard ratios for breast cancer-specific survival analysed using Cox regression for stromal CXCL14 in different breast cancer subgroups.

Immunohistochemistry does not allow determination of the cellular origin of secreted proteins within tissues. As CXCL14 is a secreted protein, we established the RNAscope 2.0 Assay that allows monitoring of CXCL14 mRNA in cells and tissue (Supplementary Figure 1). By detecting the CXCL14 mRNA, we are able to track the cell type producing the CXCL14 protein.

RNAscope is an in situ analysis technique developed for analysis of formalin-fixed paraffin-embedded tissue (FFPE) material. The partial degradation of RNA in FFPE tissues is accounted for by the use of 20 target probe pairs, spanning 40-50 nucleotides along the target RNA molecule. Furthermore, RNAscope is designed to amplify target-specific signals without amplification of background signals (Wang et al, 2012). However, pre-analytical variables including time from tumour acquisition to fixation, use of fixatives and fixation time impact on the RNA quality. The pre-analytical conditions used in this study follow standardised guidelines. However, the exact time intervals were not documented and cannot be guaranteed for all tumour samples, which is recognised as a limitation of the tumour material of this study.

Profiling of normal and tumour stroma of breast and prostate tissue has previously identified activated fibroblasts, a prominent cell type of the tumour stroma, as a source for CXCL14 (Allinen et al, 2004; Augsten et al, 2009). Compartment-specific effects of CXCL14 are suggested by findings from experimental studies. In mouse models of breast and prostate cancer, fibroblastderived CXCL14 was shown to enhance tumour growth through
NOS-dependent mechanisms that involve stimulation of angiogenesis and macrophage recruitment (Augsten et al, 2009, 2014). In contrast, anti-tumoral effects of CXCL14 have been observed in model systems where expression in malignant cells has been manipulated (Gu et al, 2012). Notably, epithelial CXCL14 expression showed a significant association with decreased proliferation in the breast cancer samples analysed in the present study (Table 1). These types of context-dependent effects have also been described for other chemokines including CCL5 and CXCL12 in model systems (Orimo et al, 2005; Wendt et al, 2008; Kojima et al, 2010; Aldinucci and Colombatti, 2014). Tumour cell expression of CXCL12 showed tumour inhibiting activities in breast cancer model systems, whereas CXCL12 from fibroblasts promoted breast tumour growth in vivo by mechanisms that involved modulation of a tumour promoting stroma (Orimo et al, 2005; Wendt et al, 2008; Kojima et al, 2010). Notably, the prognostic impact of chemokines and their receptors has also been shown to vary depending on the cell types that they are expressed in (Ohri et al, 2010).

In the present study, CXCL14 expression showed no association with tumour characteristics used to predict outcome or treatment response, which indicates that stromal CXCL14 adds additional value in defining a subgroup of breast cancer patients with poor prognosis. This notion is further supported by the finding that stroma-derived CXCL14 is a prognostic factor for breast cancer independently of, for example, tumour stage, ER $\alpha$ - status and presence of lymph-node metastasis in multivariable analysis. In addition, stromal CXCL14 expression was not associated with the abundance of tumour stroma, suggesting that the type of tumour stroma rather than the amount of stroma is important for breast cancer survival. In concordance, other marker proteins, including $\alpha$ SMA, PDGFR $\beta$, FAP, Caveolin-1, and CAF-gene expression signatures that define the type of tumour stroma have been shown to impact on breast cancer survival (Ariga et al, 2001; Surowiak et al, 2007; Finak et al, 2008; Paulsson et al, 2009; Goetz et al, 2011).

Poor survival of breast cancer is associated with the development of metastasis. The roles of CAF-derived CXCL14 during the biological processes related to metastasis formation, including tumour cell epithelial-to-mesenchymal transition (EMT), migration and invasion, might explain the correlation between stromal CXCL14 and worse breast cancer survival and should be further explored. Furthermore, in the present study the prognostic significance of stromal CXCL14 is more apparent in the ER $\alpha$ negative- and triple negative subgroups, suggesting particular relevance for stromal CXCL14 in the progression of these breast cancer subsets. Thus, it will be important to identify the inducer of CXCL14 in these breast cancer subsets, and to further explore the consequences of stromal CXCL14 expression with regard to tumour growth and metastasis. These findings should also prompt therapyoriented studies, since development of new treatments for these patients is especially urgent due to their lack of sensitivity to hormonal and HER2-directed therapies. CXCL14 should be further explored as a potential target for breast cancer treatment in patients with a prominent CXCL14 expression in the tumour stroma.

Collectively, the findings of the present study should encourage additional studies on the potential of CXCL14 as a breast cancer biomarker. These should be designed to further explore the specific impact of CXCL14 on the natural course of the disease as well as response to different treatments, and should particularly consider the cellular source of CXCL14. The associations of stromal CXCL14 with poor prognosis also suggest additional mechanistic studies where identification of the CXCL14 receptor appears as a prioritised topic. 
Table 2. Multivariable analysis for survival data, including stromal CXCL14 expression

\begin{tabular}{|c|c|c|c|c|c|c|}
\hline \multirow[t]{2}{*}{ Number (CXCL14 data) } & \multicolumn{6}{|c|}{$N=498(394)$} \\
\hline & HR & $95 \% \mathrm{Cl}$ & $P$-value & HR & $95 \% \mathrm{Cl}$ & $P$-value \\
\hline $\begin{array}{l}\leqslant 50 \\
>50\end{array}$ & $\begin{array}{l}1 \\
0.762\end{array}$ & $0.411-1.412$ & 0.388 & $\begin{array}{l}1 \\
0.731\end{array}$ & $0.451-1.184$ & 0.203 \\
\hline \multicolumn{7}{|l|}{ NHG } \\
\hline $\begin{array}{l}\text { I } \\
\text { II } \\
\text { III }\end{array}$ & $\begin{array}{l}1 \\
2.289 \\
5.645\end{array}$ & $\begin{array}{l}0.843-6.214 \\
2.139-14.900\end{array}$ & $\begin{aligned} & 0.104 \\
< & 0.0001^{*}\end{aligned}$ & $\begin{array}{l}1 \\
2.948 \\
5.427\end{array}$ & $\begin{array}{l}1.457-5.966 \\
2.680-10.990\end{array}$ & $\begin{array}{l}0.003^{*} \\
<0.0001^{*}\end{array}$ \\
\hline \multicolumn{7}{|l|}{ Node status } \\
\hline \multicolumn{7}{|l|}{ HER2 status } \\
\hline $\begin{array}{l}0 \\
1 \\
2 \\
3\end{array}$ & $\begin{array}{l}1 \\
1.393 \\
1.005 \\
0.774\end{array}$ & $\begin{array}{l}0.782-2.479 \\
0.422-2.398 \\
0.336-1.787\end{array}$ & $\begin{array}{l}0.260 \\
0.990 \\
0.549\end{array}$ & $\begin{array}{l}1 \\
1.461 \\
1.206 \\
0.706\end{array}$ & $\begin{array}{l}0.933-2.289 \\
0.623-2.335 \\
0.347-1.439\end{array}$ & $\begin{array}{l}0.098 \\
0.578 \\
0.338\end{array}$ \\
\hline \multicolumn{7}{|l|}{ Stromal CXCL14 } \\
\hline $\begin{array}{l}\text { Negative } \\
\text { Positive }\end{array}$ & $\begin{array}{l}1 \\
1.877\end{array}$ & $1.123-3.138$ & $0.016^{*}$ & $\begin{array}{l}1 \\
1.602\end{array}$ & $1.050-2.443$ & $0.029^{*}$ \\
\hline
\end{tabular}

\section{ACKNOWLEDGEMENTS}

Members of the AÖ group are acknowledged for support and constructive criticism throughout the study, and Inger Bodin at the Department of Oncology-Pathology, Karolinska Institutet, for technical assistance. The AÖ and JB groups are supported from grants from the Swedish Research Council (STARGET network), the Breast Cancer Theme Center (BRECT) at Karolinska Institutet, Swedish Cancer Society, the research funds at Radiumhemmet and Stockholm County Council (ALF). The JB group is also supported by Knut and Alice Wallenberg grant, the KI-AstraZeneca collaboration, and the Swedish Breast Cancer Association. KJ receives support from the Swedish Research Council, the Swedish Cancer Society, the Gunnar Nilsson Cancer Foundation, the Kamprad Family Foundation, the Mrs Berta Kamprad Foundation, the Swedish Government Grant for Clinical Research, and Lund University Faculty of Medicine.

\section{CONFLICT OF INTEREST}

The authors declare no conflict of interest.

\section{REFERENCES}

Aldinucci D, Colombatti A (2014) The inflammatory chemokine CCL5 and cancer progression. Mediators Inflamm 2014: 292376.

Allinen M, Beroukhim R, Cai L, Brennan C, Lahti-Domenici J, Huang H, Porter D, Hu M, Chin L, Richardson A, Schnitt S, Sellers WR, Polyak K (2004) Molecular characterization of the tumor microenvironment in breast cancer. Cancer Cell 6: 17-32.
Ariga N, Sato E, Ohuchi N, Nagura H, Ohtani H (2001) Stromal expression of fibroblast activation protein/seprase, a cell membrane serine proteinase and gelatinase, is associated with longer survival in patients with invasive ductal carcinoma of breast. Int J Cancer 95: 67-72.

Augsten M, Hagglof C, Olsson E, Stolz C, Tsagozis P, Levchenko T, Frederick MJ, Borg A, Micke P, Egevad L, Ostman A (2009) CXCL14 is an autocrine growth factor for fibroblasts and acts as a multi-modal stimulator of prostate tumor growth. Proc Natl Acad Sci USA 106: 3414-3419.

Augsten M, Sjoberg E, Frings O, Vorrink SU, Frijhoff J, Olsson E, Borg A, Ostman A (2014) Cancer-associated fibroblasts expressing CXCL14 rely upon NOS1-derived nitric oxide signaling for their tumor-supporting properties. Cancer Res 74: 2999-3010.

Balkwill F (2004) Cancer and the chemokine network. Nat Rev Cancer 4: 540-550.

Borgquist S, Holm C, Stendahl M, Anagnostaki L, Landberg G, Jirstrom K (2008) Oestrogen receptors alpha and beta show different associations to clinicopathological parameters and their co-expression might predict a better response to endocrine treatment in breast cancer. J Clin Pathol 61: 197-203.

Chiu SH, Chen CC, Lin TH (2008) Using support vector regression to model the correlation between the clinical metastases time and gene expression profile for breast cancer. Artif Intell Med 44: 221-231.

Finak G, Bertos N, Pepin F, Sadekova S, Souleimanova M, Zhao H, Chen H, Omeroglu G, Meterissian S, Omeroglu A, Hallett M, Park M (2008) Stromal gene expression predicts clinical outcome in breast cancer. Nat Med 14: 518-527.

Goetz JG, Minguet S, Navarro-Lerida I, Lazcano JJ, Samaniego R, Calvo E, Tello M, Osteso-Ibanez T, Pellinen T, Echarri A, Cerezo A, Klein-Szanto AJ, Garcia R, Keely PJ, Sanchez-Mateos P, Cukierman E, Del Pozo MA (2011) Biomechanical remodeling of the microenvironment by stromal caveolin-1 favors tumor invasion and metastasis. Cell 146: $148-163$.

Goubran HA, Kotb RR, Stakiw J, Emara ME, Burnouf T (2014) Regulation of tumor growth and metastasis: the role of tumor microenvironment. Cancer Growth Metastasis 7: 9-18. 
Gu XL, Ou ZL, Lin FJ, Yang XL, Luo JM, Shen ZZ, Shao ZM (2012) Expression of CXCL14 and its anticancer role in breast cancer. Breast Cancer Res Treat 135: 725-735.

Hara T, Tanegashima K (2012) Pleiotropic functions of the CXC-type chemokine CXCL14 in mammals. J Biochem 151: 469-476.

Kojima Y, Acar A, Eaton EN, Mellody KT, Scheel C, Ben-Porath I, Onder TT, Wang ZC, Richardson AL, Weinberg RA, Orimo A (2010) Autocrine TGF-beta and stromal cell-derived factor-1 (SDF-1) signaling drives the evolution of tumor-promoting mammary stromal myofibroblasts. Proc Natl Acad Sci USA 107: 20009-20014.

Kurth I, Willimann K, Schaerli P, Hunziker T, Clark-Lewis I, Moser B (2001) Monocyte selectivity and tissue localization suggests a role for breast and kidney-expressed chemokine (BRAK) in macrophage development. J Exp Med 194: 855-861.

Lazennec G, Richmond A (2010) Chemokines and chemokine receptors: new insights into cancer-related inflammation. Trends Mol Med 16: 133-144.

Lin K, Zou R, Lin F, Zheng S, Shen X, Xue X (2014) Expression and effect of CXCL14 in colorectal carcinoma. Mol Med Rep 10: 1561-1568.

Ohri CM, Shikotra A, Green RH, Waller DA, Bradding P (2010) Chemokine receptor expression in tumour islets and stroma in non-small cell lung cancer. BMC Cancer 10: 172.

Orimo A, Gupta PB, Sgroi DC, Arenzana-Seisdedos F, Delaunay T, Naeem R, Carey VJ, Richardson AL, Weinberg RA (2005) Stromal fibroblasts present in invasive human breast carcinomas promote tumor growth and angiogenesis through elevated SDF-1/CXCL12 secretion. Cell 121: 335-348.

Paulsson J, Micke P (2014) Prognostic relevance of cancer-associated fibroblasts in human cancer. Semin Cancer Biol 25: 61-68.

Paulsson J, Sjoblom T, Micke P, Ponten F, Landberg G, Heldin CH, Bergh J, Brennan DJ, Jirstrom K, Ostman A (2009) Prognostic significance of stromal platelet-derived growth factor beta-receptor expression in human breast cancer. Am J Pathol 175: 334-341.

Pietras K, Ostman A (2010) Hallmarks of cancer: interactions with the tumor stroma. Exp Cell Res 316: 1324-1331.

Riester M, Wei W, Waldron L, Culhane AC, Trippa L, Oliva E, Kim SH, Michor F, Huttenhower C, Parmigiani G, Birrer MJ (2014) Risk prediction for late-stage ovarian cancer by meta-analysis of 1525 patient samples. J Natl Cancer Inst 106: pii: dju048.

Sand LG, Scotlandi K, Berghuis D, Snaar-Jagalska BE, Picci P, Schmidt T, Szuhai K, Hogendoorn PC (2015) CXCL14, CXCR7 expression and CXCR4 splice variant ratio associate with survival and metastases in Ewing sarcoma patients. Eur J Cancer 51: 2624-2633.

Schwarze SR, Luo J, Isaacs WB, Jarrard DF (2005) Modulation of CXCL14 (BRAK) expression in prostate cancer. Prostate 64: 67-74.

Shellenberger TD, Wang M, Gujrati M, Jayakumar A, Strieter RM, Burdick MD, Ioannides CG, Efferson CL, El-Naggar AK, Roberts D, Clayman GL, Frederick MJ (2004) BRAK/CXCL14 is a potent inhibitor of angiogenesis and a chemotactic factor for immature dendritic cells. Cancer Res 64: 8262-8270.
Shurin GV, Ferris RL, Tourkova IL, Perez L, Lokshin A, Balkir L, Collins B, Chatta GS, Shurin MR (2005) Loss of new chemokine CXCL14 in tumor tissue is associated with low infiltration by dendritic cells (DC), while restoration of human CXCL14 expression in tumor cells causes attraction of DC both in vitro and in vivo. J Immunol 174: 5490-5498.

Sleeman MA, Fraser JK, Murison JG, Kelly SL, Prestidge RL, Palmer DJ, Watson JD, Kumble KD (2000) B cell- and monocyte-activating chemokine (BMAC), a novel non-ELR alpha-chemokine. Int Immunol 12 677-689.

Starnes T, Rasila KK, Robertson MJ, Brahmi Z, Dahl R, Christopherson K, Hromas R (2006) The chemokine CXCL14 (BRAK) stimulates activated NK cell migration: implications for the downregulation of CXCL14 in malignancy. Exp Hematol 34: 1101-1105.

Strieter RM, Burdick MD, Mestas J, Gomperts B, Keane MP, Belperio JA (2006) Cancer CXC chemokine networks and tumour angiogenesis. Eur J Cancer 42: 768-778.

Surowiak P, Murawa D, Materna V, Maciejczyk A, Pudelko M, Ciesla S, Breborowicz J, Murawa P, Zabel M, Dietel M, Lage H (2007) Occurence of stromal myofibroblasts in the invasive ductal breast cancer tissue is an unfavourable prognostic factor. Anticancer Res 27: 2917-2924.

Tessema M, Klinge DM, Yingling CM, Do K, Van Neste L, Belinsky SA (2010) Re-expression of CXCL14, a common target for epigenetic silencing in lung cancer, induces tumor necrosis. Oncogene 29: 5159-5170.

Wang F, Flanagan J, Su N, Wang LC, Bui S, Nielson A, Wu X, Vo HT, Ma XJ, Luo Y (2012) RNAscope: a novel in situ RNA analysis platform for formalin-fixed, paraffin-embedded tissues. J Mol Diagn 14: 22-29.

Wendt MK, Cooper AN, Dwinell MB (2008) Epigenetic silencing of CXCL12 increases the metastatic potential of mammary carcinoma cells. Oncogene 27: 1461-1471.

Wente MN, Mayer C, Gaida MM, Michalski CW, Giese T, Bergmann F, Giese NA, Buchler MW, Friess H (2008) CXCL14 expression and potential function in pancreatic cancer. Cancer Lett 259: 209-217.

Williams KA, Lee M, Hu Y, Andreas J, Patel SJ, Zhang S, Chines P, Elkahloun A, Chandrasekharappa S, Gutkind JS, Molinolo AA, Crawford NP (2014) A systems genetics approach identifies CXCL14, ITGAX, and LPCAT2 as novel aggressive prostate cancer susceptibility genes. PLoS Genet 10: e1004809.

Zeng J, Yang X, Cheng L, Liu R, Lei Y, Dong D, Li F, Lau QC, Deng L, Nice EC, Xie K, Huang C (2013) Chemokine CXCL14 is associated with prognosis in patients with colorectal carcinoma after curative resection. J Transl Med 11: 6.

This work is published under the standard license to publish agreement. After 12 months the work will become freely available and the license terms will switch to a Creative Commons AttributionNonCommercial-Share Alike 4.0 Unported License.

Supplementary Information accompanies this paper on British Journal of Cancer website (http://www.nature.com/bjc) 